\title{
On the Uniform Metric in the Context of Convergence to Normality
}

\author{
C. C. Heyde
}

\section{Introduction and Results}

There are two basic parts to this paper. In the first part we suppose that $X_{i}$, $i=1,2,3, \ldots$ is a sequence of independent and identically distributed random variables. We write $S_{n}=\sum_{i=1}^{n} X_{i}, n \geqq 1$, and suppose that the variables are centered so that $E X_{i}=0$ if $E\left|X_{i}\right|<\infty$. Let $\left\{B_{n}\right\}$ with $B_{n} \rightarrow \infty,\left(B_{n+1} / B_{n}\right) \rightarrow 1$ as $n \rightarrow \infty$ be a sequence of positive constants and write

and

$$
F_{n}(x)=P\left(S_{n} \leqq B_{n} x\right)
$$

where

$$
\Delta_{n}=\sup _{-\infty<x<\infty}\left|F_{n}(x)-\Phi(x)\right|
$$

$$
\Phi(x)=\frac{1}{\sqrt{2 \pi}} \int_{-\infty}^{x} e^{-\frac{1}{2} u^{2}} d u
$$

We shall establish the following theorem.

Theorem 1. If

$$
\sum_{n=1}^{\infty} n^{-1} \Delta_{n}=\sum_{n=1}^{\infty} n^{-1} \sup _{-\infty<x<\infty}\left|F_{n}(x)-\Phi(x)\right|<\infty,
$$

then $E X_{i}^{2}<\infty$. That is, the $X_{i}$ belong to the domain of normal attraction of the normal distribution.

Remarks. (1) Note that in formulating the above theorem it has not been assumed that the $X_{i}$ belong to the domain of attraction of the normal distribution. This assumption has been made in previous work on the problem, for example in Heyde [7] where it is shown that if $\sum_{n=3}^{\infty} n^{-1}(\log \log n) \Delta_{n}<\infty$ then $E X_{i}^{2}<\infty$.

(2) It is known from results of Friedman, Katz and Koopmans [3] and Heyde [5] that if $E X_{i}^{2}=\sigma^{2}<\infty$, then the series

$$
\sum_{n=1}^{\infty} n^{-1} \sup _{-\infty<x<\infty}\left|P\left(S_{n} \leqq x \sigma \sqrt{n}\right)-\Phi(x)\right|
$$

may not converge. In fact, it will converge (Heyde [5]) if and only if

$$
E X_{i}^{2} \log \left(1+\left|X_{i}\right|\right)<\infty \text {. }
$$

7 Z. Wahrscheinlichkeitstheorie verw. Geb., Bd. 25 
However, if the normalization by $\sigma \sqrt{n}$ is replaced by that by $B_{n}=\sigma_{n} \sqrt{n}$ where

then

$$
\sigma_{n}^{2}=\int_{|x|<\sqrt{n}} x^{2} d P\left(X_{i} \leqq x\right)-\left[\int_{|x|<\sqrt{n}} x d P\left(X_{i} \leqq x\right)\right]^{2}
$$

$$
\sum_{n=1}^{\infty} n^{-1} \sup _{-\infty<x<\infty}\left|P\left(S_{n} \leqq B_{n} x\right)-\Phi(x)\right|<\infty .
$$

Theorem 1 provides a converse to this last result (which is essentially due to Friedman et al. [3]; see also Theorem A of [6]).

(3) Theorem 1 is also of interest in connection with the derivation of laws of iterated logarithm type on the basis of an appropriate rate of convergence to normality as measured by the uniform metric. For details see Section 3 of [7] and Section 5 of [6].

In the second part of this paper we shall be concerned with an inequality of Osipov and Petrov. Our setting is the same as before with a sum of independent and identically distributed random variables $X_{i}, i=1,2,3, \ldots$ which are centered so that $E X_{i}=0$ if $E\left|X_{i}\right|<\infty . F(x)$ is the distribution function of the $X_{i}$ and $\left\{C_{n}\right\}$ denotes a sequence of positive constants. We shall discuss the general inequality of Osipov and Petrov [10] (see also Feller [2]) which we specialize for the present context to give

$$
\begin{aligned}
\Delta_{n}\left(C_{n}\right)= & \sup _{x}\left|P\left(S_{n} \leqq C_{n} x\right)-\Phi(x)\right| \\
& \leqq n P\left(\left|X_{i}\right|>\tau_{n}\right)+\frac{K_{0} n \int_{|x| \leqq \tau_{n}}|x|^{3} d F(x)}{B_{n}^{3}} \\
& +\frac{n\left|\int_{|x| \leqq \tau_{n}} x d F(x)\right|}{B_{n} \sqrt{2 \pi}}+\frac{1}{2 \sqrt{2 \pi e}}\left|1-\frac{B_{n}^{2}}{C_{n}^{2}}\right| \max \left(1, \frac{B_{n}^{2}}{C_{n}^{2}}\right)=\delta_{n}\left(C_{n}\right)
\end{aligned}
$$

where $B_{n}$ is given by

$$
B_{n}^{2}=n\left\{\int_{|x| \leqq \tau_{n}} x^{2} d F(x)-\left[\int_{|x| \leqq \tau_{n}} x d F(x)\right]^{2}\right\}
$$

and we shall choose $\tau_{n}$ as $\sqrt{n}$ if $E X_{i}^{2}<\infty$ and as $B_{n}$ otherwise. $K_{0}$ is a universal constant. It will be our object to show in a variety of ways that $\delta_{n}$ is a very good bound for $\Delta_{n}$. In fact, in many cases of importance we shall show that $\Delta_{n}$ and $\delta_{n}$ have equivalent asymptotic behaviour. This exercise is of special interest since no useful lower bound for $\Delta_{n}$ has been found. We shall obtain the following results.

Theorem 2. $\Delta_{n}\left(C_{n}\right) \rightarrow 0$ if and only if $\delta_{n}\left(C_{n}\right) \rightarrow 0$.

Theorem 3. Let $0<\delta<1$. Then, the following four conditions are equivalent:
(i) $\int_{-\infty}^{\infty} x^{2} d F(x)<\infty$ and $\int_{|x|>z} x^{2} d F(x)=O\left(z^{-\delta}\right)$ as $z \rightarrow \infty$,
(ii) $\inf _{C_{n}} \Delta_{n}\left(C_{n}\right)=O\left(n^{-\delta / 2}\right)$ as $n \rightarrow \infty$,
(iii) $\Delta_{n}\left(B_{n}\right)=O\left(n^{-\delta / 2}\right) \quad$ as $n \rightarrow \infty$,
(iv) $\delta_{n}\left(B_{n}\right)=O\left(n^{-\delta / 2}\right) \quad$ as $n \rightarrow \infty$. 
Theorem 4. Let $0 \leqq \delta<1$. Then, the following four conditions are equivalent:

(i) $E\left|X_{i}\right|^{2+\delta}<\infty$,

(ii) $\sum_{1}^{\infty} n^{-1+\delta / 2} \inf _{C_{n}} \Delta_{n}\left(C_{n}\right)<\infty$,

(iii) $\sum_{1}^{\infty} n^{-1+\delta / 2} \Delta_{n}\left(B_{n}\right)<\infty$,

(iv) $\sum_{1}^{\infty} n^{-1+\delta / 2} \delta_{n}\left(B_{n}\right)<\infty$.

\section{Proof of Theorem 1}

Since $\sum n^{-1} \Delta_{n}<\infty$, it follows that there exists a sequence $\left\{n_{j}\right\}$ of integers such that $\Delta_{n_{j}} \rightarrow 0$ as $j \rightarrow \infty$ and hence the $X_{i}$ belong to the domain of partial attraction of the normal distribution. Our first major task is to show that the $X_{i}$ in fact belong to the domain of attraction of the normal distribution (i.e. that $\Delta_{n} \rightarrow 0$ as $n \rightarrow \infty$, by virtue of Polya's theorem). In order to do this we need the following lemma.

Lemma. Under the assumptions of the theorem, we can choose a subsequence $\left\{n_{i}\right\}$ of the integers such that $\left(n_{i+1} / n_{i}\right) \rightarrow 1$ and $\Delta_{n_{i}} \rightarrow 0$ as $i \rightarrow \infty$.

Proof. Let $N$ denote the set of integers $n$ for which $\Delta_{n}<[\log (n+1)]^{-1}$. N contains an infinite (countable) number of elements for if not there would be a largest, $n_{L}$, and writing $u_{n}=n \log (n+1)$,

$$
\sum_{n=n_{L}}^{\infty} n^{-1} \Delta_{n} \geqq \sum_{n=n_{L}}^{\infty} u_{n}^{-1}=\infty
$$

contradicting our assumption. Then, writing $\bar{N}$ for the complement of $N$ with respect to the positive integers, we have

$$
\infty>\sum_{n=1}^{\infty} n^{-1} \Delta_{n}>\sum_{n \in \bar{N}} n^{-1} \Delta_{n} \geqq \sum_{n \in \bar{N}} u_{n}^{-1}
$$

so that $\sum_{n \in N} u_{n}^{-1}=\infty$ since

$$
\infty=\sum_{n=1}^{\infty} u_{n}^{-1}=\sum_{n \in N} u_{n}^{-1}+\sum_{n \in N} u_{n}^{-1} .
$$

Now let $N=\left\{m_{i}, i=1,2,3, \ldots ; m_{i+1}>m_{i}\right.$ each $\left.i\right\}$ and suppose that

$$
\liminf _{i \rightarrow \infty} u_{m_{i}}^{-1} u_{m_{i+1}}>1
$$

Then, there is an $\varepsilon>0$ and an integer $I=I(\varepsilon)$ such that for all $i \geqq I$,

and hence

$$
u_{m_{i+1}}>(1+\varepsilon) u_{m_{i}}
$$

$$
\sum_{i=I}^{\infty} u_{m_{i}}^{-1}<u_{m_{I}}^{-1} \sum_{r=0}^{\infty}(1+\varepsilon)^{-r}<\infty
$$


which contradicts $\sum_{n \in N} u_{n}^{-1}=\sum_{i=1}^{\infty} u_{m_{i}}^{-1}=\infty$. We therefore must have

This implies

$$
\liminf _{i \rightarrow \infty} \frac{u_{m_{i+1}}}{u_{m_{i}}}=\liminf _{i \rightarrow \infty} \frac{m_{i+1} \log \left(1+m_{i+1}\right)}{m_{i} \log \left(1+m_{i}\right)}=1 .
$$

$$
\liminf _{i \rightarrow \infty} m_{i+1} m_{i}^{-1}=1
$$

for, since $m_{i+1}>m_{i}$,

$$
1=\liminf _{i \rightarrow \infty} \frac{m_{i+1} \log \left(1+m_{i+1}\right)}{m_{i} \log \left(1+m_{i}\right)} \geqq \liminf _{i \rightarrow \infty} \frac{m_{i+1}}{m_{i}} \geqq 1 .
$$

By virtue of (3), the required subsequence $\left\{n_{i}\right\}$ can be obtained by choosing a subsequence from $N$ for which $\left(n_{i+1} / n_{i}\right) \rightarrow 1$ holds.

We now resume the proof of the theorem. Let $F(x)=P\left(X_{i} \leqq x\right)$ and suppose that $f(t)$ is the corresponding characteristic function. Firstly we shall prove the theorem for symmetric random variables (in which case $f(t)$ is real valued and symmetric).

Since $f(t)$ is continuous and $f(0)=1$, there is some interval $[-\eta, \eta]$ in which $f(t)$ may be written as $\exp \{-A(t)\}$. Then, choosing the $\left\{n_{i}\right\}$ so that $\left(n_{i+1} / n_{i}\right) \rightarrow 1$ and $\Delta_{n_{i}} \rightarrow 0$ as $i \rightarrow \infty$ (as can be done, according to the lemma) we have

$$
\left[f\left(\frac{t}{B_{n_{i}}}\right)\right]^{n_{i}}=\exp \left\{-n_{i} A\left(\frac{t}{B_{n_{i}}}\right)\right\} \rightarrow e^{-\frac{1}{2} t^{2}}
$$

so that

$$
n_{i} A\left(t B_{n_{i}}^{-1}\right) \rightarrow \frac{1}{2} t^{2}
$$

as $i \rightarrow \infty$.

Now for fixed $u, 0<u<\eta$, let $n(u)=\min \left[n_{i}: B_{n_{i}}^{-1} \leqq u\right]$. Then, $B_{n(u)}^{-1} \leqq u<B_{n(u)-1}^{-1}$ and since $A$ is continuous, $A(t u)$ lies between $A\left(t B_{n(u)}^{-1}\right)$ and $A\left(t B_{n(u)-1}^{-1}\right)$ for $0<t \leqq 1$. But, using (4),

$$
\frac{A\left(t B_{n(u)}^{-1}\right)}{A\left(B_{n(u)-1}^{-1}\right)} \sim t^{2} \frac{n(u)-1}{n(u)} \sim t^{2}
$$

as $u \rightarrow 0$ and we have

$$
\frac{A(t u)}{A(u)} \rightarrow t^{2}
$$

as $u \rightarrow 0$. Thus, $A$ is regularly varying with exponent 2 at zero $(A(t)$ is of course symmetric in $t$ in view of the assumed symmetry of $f(t))$ and we can write

$$
A(t)=\frac{1}{2} t^{2} H(t)
$$

where $H$ is slowly varying at zero. This implies that the $X_{i}$ belong to the domain of attraction of the normal distribution rather than just its domain of partial attraction (Ibragimov and Linnik [9], Chapter 2; Ibragimov [8], Lemma 2.1).

Now introduce a function $B(t)$ defined for $z>0$ by

$$
B(t)= \begin{cases}(z-t) t e^{\frac{1}{2} t^{2}}, & 0 \leqq t \leqq z, \\ 0, & \text { otherwise, }\end{cases}
$$


and let $\widehat{B}(x)$ denote its Fourier transform,

$$
\hat{B}(x)=\int_{0}^{z} e^{i t x} B(t) d t
$$

From two integrations by parts it is readily seen that $|\hat{B}(x)|=O\left(|x|^{-2}\right)$ as $|x| \rightarrow \infty$. Now write $f_{n}(t)$ for the characteristic function corresponding to $F_{n}(x)$. Then, noting that $F_{n}(x)-\Phi(x)$ is integrable and integrating by parts in the equation

$$
f_{n}(t)-e^{-\frac{1}{2} t^{2}}=\int_{-\infty}^{\infty} e^{i t x} d\left[F_{n}(x)-\Phi(x)\right]
$$

we obtain

$$
-\frac{f_{n}(t)-e^{-\frac{1}{2} t^{2}}}{i t}=\int_{-\infty}^{\infty} e^{i t x}\left[F_{n}(x)-\Phi(x)\right] d x .
$$

Consequently, using Parseval's identity on the two pairs of Fourier transforms, we obtain

$$
\begin{aligned}
i^{-1} \int_{-\infty}^{\infty} t^{-1}\left[f_{n}(t)-e^{-\frac{1}{2} t^{2}}\right] B(t) d t & =i^{-1} \int_{0}^{z} t^{-1}\left[f_{n}(t)-e^{-\frac{1}{2} t^{2}}\right] B(t) d t \\
& =\int_{-\infty}^{\infty}\left[F_{n}(x)-\Phi(x)\right] \hat{B}(x) d x
\end{aligned}
$$

Thus, from (1) and (7),

$$
\begin{aligned}
\sum_{1}^{\infty} n^{-1} & \left|\int_{0}^{z}(z-t)\left(f_{n}(t)-e^{-\frac{1}{2} t^{2}}\right) e^{\frac{1}{2} t^{2}} d t\right| \\
& =\sum_{1}^{\infty} n^{-1}\left|\int_{-\infty}^{\infty}\left[F_{n}(x)-\Phi(x)\right] \hat{B}(x) d x\right| \\
& \leqq \sum_{1}^{\infty} n^{-1} \Delta_{n} \int_{-\infty}^{\infty}|\hat{B}(x)| d x<\infty .
\end{aligned}
$$

Now, by (5), $f(t)$ is representable in the form

$$
f(t)=\exp \left\{-\frac{1}{2} t^{2} H(t)\right\}
$$

where $H(t)$ is a slowly varying function as $t \rightarrow 0$ and moreover, by Lemma 2.1 of [8],

as $t \rightarrow 0$. Then

$$
H(t) \sim \int_{|x t| \leqq 1} x^{2} d F(x)
$$

$$
f_{n}(t)=\left[f\left(\frac{t}{B_{n}}\right)\right]^{n}=\exp \left\{-\frac{n t^{2}}{2 B_{n}^{2}} H\left(\frac{t}{B_{n}}\right)\right\}
$$

so that (8) gives

$$
\sum_{1}^{\infty} n^{-1}\left|\int_{0}^{z}(z-t)\left\{\exp \left[-\frac{t^{2}}{2}\left(\frac{n}{B_{n}^{2}} H\left(\frac{t}{B_{n}}\right)-1\right)\right]-1\right\} d t\right|<\infty .
$$


However, as $n \rightarrow \infty$,

$$
\exp \left[-\frac{t^{2}}{2}\left(\frac{n}{B_{n}^{2}} H\left(\frac{t}{B_{n}}\right)-1\right)\right]-1=\frac{t^{2}}{2}\left[1-\frac{n}{B_{n}^{2}} H\left(\frac{t}{B_{n}}\right)\right]+R_{n}(t)
$$

where

$$
R_{n}(t)=\sum_{r=2}^{\infty}\left[1-\frac{n}{B_{n}^{2}} H\left(\frac{t}{B_{n}}\right)\right]^{r} \frac{t^{2 r}}{2^{r} r !}
$$

and for $n$ sufficiently large and $0 \leqq t \leqq z$,

$$
\begin{aligned}
\left|R_{n}(t)\right| & \leqq t^{4}\left[1-\frac{n}{B_{n}^{2}} H\left(\frac{t}{B_{n}}\right)\right]^{2} \sum_{r=2}^{\infty} \frac{z^{2(r-1)}}{2^{r} r !} \\
& \leqq z^{-2} e^{\frac{1}{2} z^{2}} t^{4}\left[1-\frac{n}{B_{n}^{2}} H\left(\frac{t}{B_{n}}\right)\right]^{2}
\end{aligned}
$$

Now, $F_{n}(x)-\Phi(x)$ obviously belongs to $L_{2}(-\infty, \infty)$. In fact, $\int_{-\infty}^{\infty}\left|F_{n}(x)-\Phi(x)\right|^{\alpha} d x$ exists and $\rightarrow 0$ as $n \rightarrow \infty$ for $\alpha>\frac{1}{2}$ (see Ibragimov and Linnik [9], Theorem 5.2.1, p. 172). It then follows from the Parseval identity that

$$
\int_{-\infty}^{\infty}\left[F_{n}(x)-\Phi(x)\right]^{2} d x=\frac{1}{2 \pi} \int_{-\infty}^{\infty} t^{-2}\left[f_{n}(t)-e^{-\frac{1}{2} t^{2}}\right]^{2} d t .
$$

Furthermore,

$$
\sum_{1}^{\infty} n^{-1} \int_{-\infty}^{\infty}\left[F_{n}(x)-\Phi(x)\right]^{2} d x \leqq \sum_{1}^{\infty} n^{-1} \sup _{x}\left|F_{n}(x)-\Phi(x)\right| \int_{-\infty}^{\infty}\left|F_{n}(x)-\Phi(x)\right| d x<\infty
$$

in view of (1) so that from (12),

$$
\sum_{1}^{\infty} n^{-1} \int_{-\infty}^{\infty} t^{-2}\left[f_{n}(t)-e^{-\frac{1}{2} t^{2}}\right]^{2} d t<\infty
$$

and in particular

But, as $n \rightarrow \infty$,

$$
\sum_{1}^{\infty} n^{-1} \int_{0}^{z} t^{-2}\left[f_{n}(t)-e^{-\frac{1}{2} t^{2}}\right]^{2} d t<\infty
$$

$$
\left|\frac{f_{n}(t)-e^{-\frac{1}{2} t^{2}}}{t}\right|=\frac{1}{2} e^{-\frac{1}{2} t^{2}}|t|\left|1-\frac{n}{B_{n}^{2}} H\left(\frac{t}{B_{n}}\right)\right|(1+o(1))
$$

so that (13) gives

$$
\sum_{1}^{\infty} n^{-1} \int_{0}^{z} e^{-t^{2}} t^{2}\left[1-\frac{n}{B_{n}^{2}} H\left(\frac{t}{B_{n}}\right)\right]^{2} d t<\infty .
$$

We then deduce, using (11), that

$$
\sum_{1}^{\infty} n^{-1} \int_{0}^{z}(z-t)\left|R_{n}(t)\right| d t<\infty
$$


and, from (9) and (10),

$$
\sum_{1}^{\infty} n^{-1}\left|\int_{0}^{z}(z-t) t^{2}\left[1-\frac{n}{B_{n}^{2}} H\left(\frac{t}{B_{n}}\right)\right] d t\right|<\infty .
$$

Now make the transformation $t=z x$; we obtain

$$
\sum_{1}^{\infty} n^{-1}\left|\int_{0}^{1}(1-x) x^{2}\left[1-\frac{n}{B_{n}^{2}} H\left(\frac{z x}{B_{n}}\right)\right] d x\right|<\infty .
$$

Therefore, from (14),

or equivalently,

$$
\begin{aligned}
\sum_{1}^{\infty} \frac{1}{B_{n}^{2}} & \left|\int_{0}^{1}(1-x) x^{2}\left[H\left(\frac{x}{B_{n}}\right)-H\left(\frac{2 x}{B_{n}}\right)\right] d x\right| \\
\leqq & \sum_{1}^{\infty} n^{-1}\left|\int_{0}^{1}(1-x) x^{2}\left[1-\frac{n}{B_{n}^{2}} H\left(\frac{2 x}{B_{n}}\right)\right] d x\right| \\
& +\sum_{1}^{\infty} n^{-1}\left|\int_{0}^{1}(1-x) x^{2}\left[1-\frac{n}{B_{n}^{2}} H\left(\frac{x}{B_{n}}\right)\right] d x\right|<\infty,
\end{aligned}
$$

Now,

$$
\sum_{1}^{\infty}\left|\int_{0}^{1}(1-x)\left[\log f\left(2 B_{n}^{-1} x\right)-4 \log f\left(B_{n}^{-1} x\right)\right] d x\right|<\infty .
$$

where

$$
\begin{aligned}
\log f & \left(2 B_{n}^{-1} x\right)-4 \log f\left(B_{n}^{-1} x\right) \\
& =\log \left[1-\left\{1-f\left(2 B_{n}^{-1} x\right)\right\}\right]-4 \log \left[1-\left\{1-f\left(B_{n}^{-1} x\right)\right\}\right] \\
& =-\left\{1-f\left(2 B_{n}^{-1} x\right)\right\}+4\left\{1-f\left(B_{n}^{-1} x\right)\right\}+C_{n}(x)
\end{aligned}
$$

$$
C_{n}(x)=\sum_{r=2}^{\infty} r^{-1}\left[4\left\{1-f\left(B_{n}^{-1} x\right)\right\}^{r}-\left\{1-f\left(2 B_{n}^{-1} x\right)\right\}^{r}\right] .
$$

Furthermore, as $n \rightarrow \infty$ we have for $0<x \leqq 1$,

$$
\begin{aligned}
& 1-f\left(2 B_{n}^{-1} x\right) \sim \frac{1}{2}\left(2 B_{n}^{-1} x\right)^{2} H\left(2 B_{n}^{-1} x\right) \sim 2 n^{-1} x^{2} \\
& 1-f\left(B_{n}^{-1} x\right) \sim \frac{1}{2}\left(B_{n}^{-1} x\right)^{2} H\left(B_{n}^{-1} x\right) \sim \frac{1}{2} n^{-1} x^{2},
\end{aligned}
$$

while $C_{n}(0)=0$, so that

$$
\sum_{1}^{\infty} \int_{0}^{1}(1-x)\left|C_{n}(x)\right| d x<\infty
$$

Consequently, from (15) and (16),

$$
\sum_{1}^{\infty}\left|\int_{0}^{1}(1-x)\left[4\left\{1-f\left(B_{n}^{-1} x\right)\right\}-\left\{1-f\left(2 B_{n}^{-1} x\right)\right\}\right] d x\right|<\infty .
$$

This may be rewritten as (remembering that $f(t)$ is real valued)

$$
\sum_{1}^{\infty}\left|\int_{0}^{1}(1-x)\left[\int_{-\infty}^{\infty}\left\{4\left(1-\cos B_{n}^{-1} x y\right)-\left(1-\cos 2 B_{n}^{-1} x y\right)\right\} d F(y)\right] d x\right|<\infty,
$$


which reduces to

and hence

$$
\sum_{1}^{\infty} \int_{0}^{1}(1-x)\left\{\int_{-\infty}^{\infty}\left(1-\cos B_{n}^{-1} x y\right)^{2} d F(y)\right\} d x<\infty
$$

$$
\sum_{1}^{\infty} \int_{0}^{1}(1-x)\left\{\int_{|y| \leqq \pi B_{n}}\left(1-\cos B_{n}^{-1} x y\right)^{2} d F(y)\right\} d x<\infty .
$$

Now, for $|y| \leqq \pi B_{n}$, we can find a positive constant $C$ so that

$$
1-\cos B_{n}^{-1} x y \geqq C\left(B_{n}^{-1} x y\right)^{2}, \quad 0 \leqq x \leqq 1,
$$

and therefore,

that is,

$$
\sum_{1}^{\infty} B_{n}^{-4} \int_{0}^{1} x^{4}(1-x)\left\{\int_{|y| \leqq \pi B_{n}} y^{4} d F(y)\right\} d x<\infty,
$$

$$
\sum_{1}^{\infty} B_{n}^{-4} \int_{|y| \leqq \pi B_{n}} y^{4} d F(y)<\infty .
$$

$\left\{B_{n}\right\}$ has not been assumed monotone but we can now make this supposition in (17) without loss of generality (since we may discard terms and relabel the remaining ones if necessary). (17) is then Eq. (10) of Heyde [6] and following exactly the argument given in the remainder of Section 2 of [6] we obtain the required result that $E X_{i}^{2}<\infty$.

Finally, suppose that the $X_{i}$ are not symmetric. We consider the sequence $Y_{i}$, $i=1,2,3, \ldots$ of independent and symmetric random variables, each $Y_{i}$ having the distribution of the difference between two independent $X_{i}$ 's. Obviously the characteristic function of $Y_{i}$ is $|f(t)|^{2}$ and the distribution function of the sum $Z_{n}=\left(Y_{1}+\cdots+Y_{n}\right) / B_{n} \sqrt{2}$ is equal to $F_{n}(x \sqrt{2}) *\left[1-F_{n}(-x \sqrt{2}-0)\right]=G_{n}(x)$. Hence, if $F_{n}(x)$ satisfies the condition (1), we have

$$
\begin{aligned}
\sum_{1}^{\infty} n^{-1} & \sup _{x}\left|G_{n}(x)-\Phi(x)\right| \\
= & \sum_{1}^{\infty} n^{-1} \sup _{x}\left|F_{n}(x \sqrt{2}) *\left(1-F_{n}(-x \sqrt{2}-0)\right)-\Phi(x \sqrt{2}) *(1-\Phi(-x \sqrt{2}))\right| \\
& \leqq \\
& \sum_{1}^{\infty} n^{-1} \sup _{x}\left|F_{n}(x \sqrt{2}) *\left(1-F_{n}(-x \sqrt{2}-0)\right)-\Phi(x \sqrt{2}) *\left(1-F_{n}(-x \sqrt{2}-0)\right)\right| \\
& +\sum_{1}^{\infty} n^{-1} \sup _{x}\left|\Phi(x \sqrt{2}) *\left(1-F_{n}(-x \sqrt{2}-0)\right)-\Phi(x \sqrt{2}) *(1-\Phi(-x \sqrt{2}))\right| \\
<\infty &
\end{aligned}
$$

Then, from our results in the symmetric case, we extract the information that $E Y_{i}^{2}<\infty$ from which it follows that $E X_{i}^{2}<\infty$. This completes the proof of the theorem. 


\section{Proofs of Theorems 2, 3, 4}

Proof of Theorem 2. That $\Delta_{n}\left(C_{n}\right) \rightarrow 0$ if $\delta_{n}\left(C_{n}\right) \rightarrow 0$ follows from (2) so we suppose $\Delta_{n}\left(C_{n}\right) \rightarrow 0$ (i. e. the $X_{i}$ belong to the domain of attraction of the normal distribution) and need to show that $\delta_{n}\left(C_{n}\right) \rightarrow 0$ in order to complete the proof.

Now, as already noted in the proof of Theorem 1, we have that $U(x)=$ $\int_{|u| \leqq x} u^{2} d F(u)$ is slowly varying as $x \rightarrow \infty$. Clearly $n B_{n}^{-2} U\left(B_{n}\right) \rightarrow 1$ and $B_{n} \sim C_{n}$ as $|u| \leqq x$
$n \rightarrow \infty$ . Also, from Gnedenko and Kolmogorov [4] p. 172 we have that

$$
x^{2} P\left(\left|X_{i}\right|>x\right) / U(x) \rightarrow 0
$$

as $x \rightarrow \infty$.

If $E X_{i}^{2}<\infty$ we have $\tau_{n}=\sqrt{n}$ and $n P\left(\left|X_{1}\right|>\sqrt{n}\right) \rightarrow 0$ since the terms of

$$
\sum P\left(\left|X_{i}\right|>\sqrt{n}\right)<\infty
$$

are monotone. If $E X_{i}^{2}=\infty$ we have $\tau_{n}=B_{n}$ and from (18),

$$
n P\left(\left|X_{i}\right|>B_{n}\right) \sim B_{n}^{2}\left[U\left(B_{n}\right)\right]^{-1} P\left(\left|X_{i}\right|>B_{n}\right) \rightarrow 0
$$

as $n \rightarrow \infty$. In either case,

$$
n P\left(\left|X_{i}\right|>\tau_{n}\right) \rightarrow 0 \quad \text { as } n \rightarrow \infty .
$$

Next, we have

$$
\begin{aligned}
n B_{n}^{-3} \int_{|x| \leqq \tau_{n}}|x|^{3} d F(x) & =n B_{n}^{-3} \int_{0}^{\tau_{n}} x d U(x) \\
& =n \tau_{n} B_{n}^{-3} U\left(\tau_{n}\right)-n B_{n}^{-3} \int_{0}^{\tau_{n}} U(x) d x
\end{aligned}
$$

and $n B_{n}^{-3} \int_{0}^{\tau_{n}} U(x) d x \sim n \tau_{n} B_{n}^{-3} U\left(\tau_{n}\right)$ using Theorem 1, p. 273 of Feller [1]. If $E X_{i}^{2}<\infty$ we have $\tau_{n}=\sqrt{n}, B_{n} \sim\left(n E X_{i}^{2}\right)^{\frac{1}{2}}$ and $U\left(\tau_{n}\right) \rightarrow E X_{i}^{2}$ as $n \rightarrow \infty$ while if $E X_{i}^{2}=\infty$ we have $\tau_{n}=B_{n}$ and $n B_{n}^{-2} U\left(B_{n}\right) \rightarrow 1$. In either case,

$$
n B_{n}^{-3} \int_{|x| \leqq \tau_{n}}|x|^{3} d F(x) \rightarrow 0 \quad \text { as } n \rightarrow \infty .
$$

Finally, we have

$$
\begin{aligned}
n B_{n}^{-1}\left|\int_{|x| \leqq \tau_{n}} x d F(x)\right| & =n B_{n}^{-1}\left|\int_{|x|>\tau_{n}} x d F(x)\right| \\
& \leqq n B_{n}^{-1} \int_{|x|>\tau_{n}}|x| d F(x) \\
& \leqq n B_{n}^{-1} \tau_{n} P\left(\left|X_{i}\right|>\tau_{n}\right)+n B_{n}^{-1} \int_{\tau_{n}}^{\infty} P\left(\left|X_{i}\right|>x\right) d x .
\end{aligned}
$$

The first term on the right hand side of (21) goes to zero as $n \rightarrow \infty$ by (19). To deal with the second term we use (18) and, given any $\varepsilon>0$, choose $N=N(\varepsilon)$ so large that

$$
P\left(\left|X_{i}\right|>x\right) \leqq \varepsilon x^{-2} U(x)
$$


for $x \geqq N$. Consequently, for $n$ sufficiently large that $\tau_{n}>N$,

$$
n B_{n}^{-1} \int_{\tau_{n}}^{\infty} P\left(\left|X_{i}\right|>x\right) d x \leqq \varepsilon n B_{n}^{-1} \int_{\tau_{n}}^{\infty} x^{-2} U(x) d x .
$$

Another application of Theorem 1, p. 273 of [1] gives

$$
n B_{n}^{-1} \int_{\tau_{n}}^{\infty} x^{-2} U(x) d x \sim n B_{n}^{-1} \tau_{n}^{-1} U\left(\tau_{n}\right) \rightarrow\left(E X_{i}^{2}\right)^{\frac{1}{2}} \quad \text { if } E X_{i}^{2}<\infty, 1
$$

otherwise as $n \rightarrow \infty$ since either $B_{n} \sim\left(n E X_{i}^{2}\right)^{\frac{1}{2}}, U\left(\tau_{n}\right) \rightarrow E X_{i}^{2}, \tau_{n}=\sqrt{n}$ in case $E X_{i}^{2}<\infty$ or $\tau_{n}=B_{n}, n B_{n}^{-2} U\left(B_{n}\right) \rightarrow 1$ in case $E X_{i}^{2}=\infty$. Thus, from (21),

$$
n B_{n}^{-1}\left|\int_{|x| \leqq \tau_{n}} x d F(x)\right| \rightarrow 0 \quad \text { as } n \rightarrow \infty .
$$

The required result $\delta_{n}\left(C_{n}\right) \rightarrow 0$ follows from (19), (20) and (22), since $C_{n} \sim B_{n}$ as $n \rightarrow \infty$.

Proof of Theorem 3. The equivalence of (i) and (ii) follows from Theorem 3.1 of Ibragimov [8]. We thus have (iv) $\Rightarrow$ (iii) $\Rightarrow$ (ii) $\Rightarrow$ (i) and to complete the proof it is just necessary to show that (i) $\Rightarrow$ (iv).

Now, when (i) holds we have

$$
\begin{aligned}
\left|\int_{|x| \leqq \sqrt{n}} x d F(x)\right| & =\left|\int_{|x|>\sqrt{n}} x d F(x)\right| \\
& \leqq \int_{|x|>\sqrt{n}}|x| d F(x) \\
& \leqq n^{-\frac{1}{2}} \int_{|x|>\sqrt{n}} x^{2} d F(x)=O\left(n^{-(1+\delta) / 2}\right)
\end{aligned}
$$

and $B_{n}^{2} \sim n E X_{i}^{2}$ as $n \rightarrow \infty$ so that

$$
n B_{n}^{-1}\left|\int_{|x| \leqq \sqrt{n}} x d F(x)\right|=O\left(n^{-\delta / 2}\right) .
$$

Also, putting $R(z)=\int_{|u|>z} u^{2} d F(u)$, we have

$$
\begin{aligned}
\int_{|x| \leqq \sqrt{n}}|x|^{3} d F(x) & =-\int_{0}^{\sqrt{n}} u d R(u) \\
& =-\sqrt{n} R(\sqrt{n})+\int_{0}^{\sqrt{n}} R(u) d u=O\left(n^{(1-\delta) / 2}\right)
\end{aligned}
$$

so that

$$
B_{n}^{-3} n \int_{|x| \leqq \sqrt{n}}|x|^{3} d F(x)=O\left(n^{-\delta / 2}\right) .
$$

Finally,

$$
n P\left(\left|X_{i}\right|>\sqrt{n}\right) \leqq \int_{|x|>\sqrt{n}} x^{2} d F(x)=O\left(n^{-\delta / 2}\right),
$$

and (iv) follows from (23), (24) and (25). 
Proof of Theorem 4. If $E X_{i}^{2}<\infty$, the equivalence of (i) and (iii) follows easily from results given in Friedman, Katz and Koopmans [3]. That (iii) implies $E X_{i}^{2}<\infty$ follows from Theorem 1 . We also have (iv) $\Rightarrow$ (iii) $\Rightarrow$ (ii) so it remains to prove that (i) $\Rightarrow$ (iv) and (ii) $\Rightarrow$ (i).

First we shall prove that (i) $\Rightarrow$ (iv). We have

$$
\sum_{1}^{\infty} n^{\delta / 2} P\left(\left|X_{i}\right|>\sqrt{n}\right)<\infty
$$

since $E\left|X_{i}\right|^{2+\delta}<\infty$. Also, $B_{n} \sim\left(n E X_{i}^{2}\right)^{\frac{1}{2}}$ as $n \rightarrow \infty$ and using integration by parts,

$$
\begin{aligned}
& \sum_{n=1}^{\infty} n^{(\delta-3) / 2} \int_{|x| \leqq \sqrt{n}}|x|^{3} d F(x) \\
& \quad \leqq 3 \sum_{n=1}^{\infty} n^{(\delta-3) / 2} \sum_{k=1}^{n} \int_{\sqrt{k-1}<x \leqq \sqrt{k}} x^{2} P\left(\left|X_{i}\right|>x\right) d x \\
& \quad \leqq \sum_{n=1}^{\infty} n^{(\delta-3) / 2} \sum_{k=1}^{n} P\left(\left|X_{i}\right|>\sqrt{k-1}\right)\left[k^{\frac{3}{2}}-(k-1)^{\frac{3}{2}}\right] \\
& \quad \leqq C_{1} \sum_{k=1}^{\infty} P\left(\left|X_{i}\right|>\sqrt{k-1}\right) k^{\frac{1}{2}} \sum_{n=k}^{\infty} n^{(\delta-3) / 2} \\
& \leqq C_{2} \sum_{k=1}^{\infty} k^{\delta / 2} P\left(\left|X_{i}\right|>\sqrt{k-1}\right)<\infty
\end{aligned}
$$

$C_{1}, C_{2}$ denoting suitable positive constants. Furthermore,

$$
\begin{aligned}
\sum_{n=1}^{\infty} n^{(\delta-1) / 2}\left|\int_{|x| \leqq \sqrt{n}} x d F(x)\right| \\
\quad \leqq \sum_{n=1}^{\infty} n^{(\delta-1) / 2} \int_{|x|>\sqrt{n}}|x| d F(x) \\
\quad=\sum_{n=1}^{\infty} n^{(\delta-1) / 2}\left\{\sqrt{n} P\left(\left|X_{i}\right|>\sqrt{n}\right)+\int_{\sqrt{n}}^{\infty} P\left(\left|X_{i}\right|>x\right) d x\right\} \\
\quad=\sum_{n=1}^{\infty} n^{\delta / 2} P\left(\left|X_{i}\right|>\sqrt{n}\right)+\sum_{n=1}^{\infty} n^{(\delta-1) / 2} \sum_{k=n}^{\infty} \int_{\sqrt{k}<x \leqq \sqrt{k+1}} P\left(\left|X_{i}\right|>x\right) d x \\
\quad \leqq \sum_{n=1}^{\infty} n^{\delta / 2} P\left(\left|X_{i}\right|>\sqrt{n}\right)+\sum_{n=1}^{\infty} n^{(\delta-1) / 2} \sum_{k=n}^{\infty} P\left(\left|X_{i}\right|>\sqrt{k}\right)(\sqrt{k+1}-\sqrt{k}) \\
\quad \leqq \sum_{n=1}^{\infty} n^{\delta / 2} P\left(\left|X_{i}\right|>\sqrt{n}\right)+D_{1} \sum_{k=1}^{\infty} P\left(\left|X_{i}\right|>\sqrt{k}\right) k^{-\frac{1}{2}} \sum_{n=1}^{k} n^{(\delta-1) / 2} \\
\leqq \\
\quad D_{2} \sum_{k=1}^{\infty} k^{\delta / 2} P\left(\left|X_{i}\right|>\sqrt{k}\right)<\infty
\end{aligned}
$$

$D_{1}, D_{2}$ denoting suitable positive constants. (iv) follows from (26), (27), (28). 
Finally, we need to prove that (ii) $\Rightarrow$ (i). Choose $\left\{C_{n}^{*}\right\}$ so that $\Delta_{n}\left(C_{n}^{*}\right) \sim \inf _{C_{n}} \Delta_{n}\left(C_{n}\right)$ and fix attention on $\sum n^{-1+\delta / 2} \Delta_{n}\left(C_{n}^{*}\right)<\infty$. That (i) holds for $\delta=0$ is given by Theorem 1 and the proof for $\delta>0$ is obtained by paralleling the proof of Theorem 1 . Instead of (8) we now have

$$
\sum_{1}^{\infty} n^{-1+\delta / 2}\left|\int_{0}^{z}(z-t)\left(f_{n}(t)-e^{-\frac{1}{2} t^{2}}\right) e^{\frac{1}{2} t^{2}} d t\right|<\infty,
$$

where

and

$$
f_{n}(t)=\left[f\left(t / C_{n}^{*}\right)\right]^{n}
$$

$$
f(t)=\exp \left\{-\frac{1}{2} t^{2} H(t)\right\}
$$

with $H(t) \rightarrow E X_{i}^{2}$ as $t \rightarrow 0$. The proof of Theorem 1 can then be followed through in similar fashion up to the stage of obtaining

$$
\sum_{1}^{\infty} n^{\delta / 2} \int_{0}^{1}(1-x)\left\{\int_{|y| \leqq \sigma \sqrt{n}}\left(1-\cos \left(C_{n}^{*}\right)^{-1} x y\right)^{2} d F(y)\right\} d x<\infty
$$

where $\sigma^{2}=E X_{i}^{2}$. Now, noting that $C_{n}^{*} \sim\left(n E X_{i}^{2}\right)^{\frac{1}{2}}$ as $n \rightarrow \infty$ we can, for $|y| \leqq \pi C_{n}^{*}$, find a positive constant $K$ such that

$$
1-\cos \left(C_{n}^{*}\right)^{-1} x y \geqq K\left(n^{-\frac{1}{2}} x\right)^{2}, \quad 0 \leqq x \leqq 1,
$$

and therefore (29) yields

$$
\sum_{1}^{\infty} n^{(\delta / 2)-2} \int_{|y| \leqq \sigma \sqrt{n}} y^{4} d F(y)<\infty .
$$

Furthermore,

$$
\begin{aligned}
\sum_{n=1}^{\infty} n^{(\delta / 2)-2} \int_{|x| \leqq \sigma \sqrt{n}} x^{4} d F(x) & \geqq \sum_{n=2}^{\infty} n^{(\delta / 2)-2} \sum_{k=1}^{n-1} \int_{\sigma \sqrt{k}<|x| \leqq \sigma \sqrt{k+1}} x^{4} d F(x) \\
& \geqq \sigma^{4} \sum_{k=1}^{\infty} k^{2} P\left(\sigma \sqrt{k}<\left|X_{i}\right| \leqq \sigma \sqrt{k+1}\right) \sum_{n=k+1}^{\infty} n^{(\delta / 2)-2} \\
& \geqq C \sum_{k=1}^{\infty} k^{1+\delta / 2} P\left(\sigma \sqrt{k}<\left|X_{i}\right| \leqq \sigma \sqrt{k+1}\right)
\end{aligned}
$$

for some positive constant $C$, while

$$
\begin{aligned}
E\left|X_{i}\right|^{2+\delta} & =\sum_{k=0}^{\infty} \int_{\sigma \sqrt{k}<|x| \leqq \sigma \sqrt{k+1}}|x|^{2+\delta} d F(x) \\
& \leqq C_{1} \sum_{k=1}^{\infty} k^{1+\delta / 2} P\left(\sigma \sqrt{k}<\left|X_{i}\right| \leqq \sigma \sqrt{k+1}\right)
\end{aligned}
$$

for some positive constant $C_{1}$ and hence $E\left|X_{i}\right|^{2+\delta}<\infty$. This proves the required result (i) for symmetric random variables; the result for the general case is obtained as in the proof of Theorem 1. 


\section{References}

1. Feller, W.: An introduction to probability theory and its applications, Vol. II. New York: Wiley 1966.

2. Feller, W.: On the Berry-Esseen theorem. Z. Wahrscheinlichkeitstheorie verw. Gebiete 10, 261-268 (1968).

3. Friedman, N., Katz, M., Koopmans, L.H.: Convergence rates for the central limit theorem. Proc. Nat. Acad. Sci. U.S. A. 56, 1062-1065 (1966).

4. Gnedenko, B.V. and A. N. Kolmogorov: Limit distributions for sums of independent random variables. Reading, Mass.: Addison-Wesley 1954.

5. Heyde, C.C.: On the influence of moments on the rate of convergence to the normal distribution. Z. Wahrscheinlichkeitstheorie verw. Gebiete 8, 12-18 (1967).

6. Heyde, C.C.: Some properties of metrics in a study on convergence to normality. Z. Wahrscheinlichkeitstheorie verw. Gebiete 11, 181-192 (1969).

7. Heyde, C.C.: On the implication of a certain rate of convergence to normality. Z. Wahrscheinlichkeitstheorie verw. Gebiete 16, 151-156 (1970).

8. Ibragimov, I.A.: On the accuracy of the Gaussian approximation to the distribution function of sums of independent random variables. Theor. Probab. Appl. 11, 559-576 (1966).

9. Ibragimov, I.A. and Yu. V. Linnik: Independent and stationary dependent random variables (in Russian). Moscow Izd-vo "Nauka" 1965.

10. Osipov, L.V. and V.V. Petrov: On an estimate of the remainder in the central limit theorem. Theor. Probab. Appl. 12, 281-286 (1967).

C.C. Heyde

Department of Statistics, SGS

Australian National University

Box 4, P.O.

Canberra, A.C.T. 2600

Australia

(Received December 16,1971) 